BuLLETIN Bulletin hispanique

HISPANIQUE Université Michel de Montaigne Bordeaux

112-1| 2010

Actes du Colloque « langue, littérature, littéralité »

\title{
De Circé à Ulysse
}

le naufragé « gothique » de Lope de Vega

\section{Maria Aranda}

\section{(2) OpenEdition}

Journals

Édition électronique

URL : http://journals.openedition.org/bulletinhispanique/1092

DOI : 10.4000/bulletinhispanique.1092

ISSN : 1775-3821

Éditeur

Presses universitaires de Bordeaux

\section{Édition imprimée}

Date de publication : 1 juin 2010

Pagination : 61-73

ISBN : 978-2-86781-692-5

ISSN : 0007-4640

Référence électronique

Maria Aranda, «De Circé à Ulysse », Bulletin hispanique [En ligne], 112-1 | 2010, mis en ligne le 01 juin 2013, consulté le 20 avril 2019. URL : http://journals.openedition.org/bulletinhispanique/1092 ; DOI : 10.4000/bulletinhispanique.1092 


\title{
De Circé à Ulysse : le naufragé « gothique » de Lope de Vega
}

\author{
Maria Aranda \\ Université Michel de Montaigne Bordeaux
}

En hommage et en (modeste) complément à l'étude consacrée par Nadine Ly à l'architecture de La Circe de Lope de Vega, cette nouvelle approche sintéresse au héros masculin à travers l'analyse littérale de textes choisis (un sonnet extrait des Rimas; la célèbre tragi-comédie El Caballero de Olmedo). Par-delà le traditionnel peregrino amoroso, le poète-dramaturge invente une écriture du fantastique et de la noirceur "gothiques".

En homenaje y (modesto) complemento al estudio dedicado por Nadine Ly a la arquitectura de La Circe de Lope de Vega, esta nueva propuesta se centra en el héroe masculino a través del análisis literal de textos selectos (un soneto sacado de las Rimas; la famosa tragicomedia de El Caballero de Olmedo). Más allá del tradicional peregrino amoroso, el poeta-dramaturgo inventa una escritura de lo fantástico y del tenebrismo "góticos".

As a tribute and (modest) complement to Nadine Ly's study, dedicated to the architecture of Lope de Vega's La Circe, this new approach is interested in the masculine hero, through a literal analysis of selected writings (a sonnet from the Rimas', as well as the famous tragi-comedia : 'El Caballero de Olmedo'). Beyond the traditional peregrino amoroso, the poet and dramatist explores what inventing 'gothic' fantasy and darkness means.

Mots-clés : Lope de Vega - El Caballero de Olmedo - Peregrino amoroso - littérature gothique - analyse littérale.

$B H i$, Tome 112, nº 1 - juin 2010 - p. 61 à 73. 
$\mathrm{L}$

E POINT de départ de cette étude est le souvenir très fort laissé dans ma mémoire par les travaux de Nadine Ly sur La Circe de Lope de Vega. Je me rends compte à présent qu'il n'est peut-être pas fortuit que, d'emblée, se soit imposée à moi la figure de la magicienne, à la fois personnage tutélaire et principe poétique. Bien d'autres moments exceptionnels de découverte auraient pu donner matière à une recherche-hommage, mais c'est la mise au jour de l'architecture de cette si difficile œuvre miscellanée que je retiens, car elle continue d'imprégner toutes mes lectures lopesques, et que je crois apercevoir dans chaque texte, par transparence, les cercles de Circé et ses transmutations littérales.

L'on se souvient que l'accomplissement de cette " Euvre au Blanc" " que le texte de 1624 constitue est soumis à l'audacieuse "spiritualisation ou à la désexualisation des rapports entre Circé et Ulysse ${ }^{2}$ ». Ce processus de métamorphose et d'abstraction, s'il implique une double solitude purificatrice de la magicienne et du naufragé mythique, sous-entend également l'expérience des ténèbres et le dédale de l'errance. C'est à l'autre versant de l'écriture poétique de Lope - non plus celle qui est apte aux opérations alchimiques de l'albédo, mais son envers plus sombre - que seront consacrées ces remarques.

La puissante empreinte odysséenne que l'on observe dans l'œuvre du dramaturge s'est facilement combinée, on le sait, à d'autres influences proprement lyriques. Les naufrages répétés d'Ulysse - personnage au centre d'une sorte d'autofiction spectrale qui informe, peut-être, la production entière du Phénix - apparaissent, par exemple, dans les Rimas de 1602, confondus avec le motif pétrarquiste du peregrino amoroso. Les sonnets consacrés par l'auteur (sur le modèle du maître italien) au parcours désolé du sujet à travers une Nature sombre et déserte, ont été abondamment étudiés ${ }^{3}$. Mais il en est un en particulier, le sonnet 19, qui, malgré son inscription flagrante dans la tradition de l'homo viator, s'en distingue par l'irréalisme que l'on dirait onirique de ses figures ${ }^{4}$.

1. Nadine Ly, "La Circe de Lope de Vega, ou la "conversation" d'Ulysse et de Circé : étymologie et littéralité structurale ", Littéralité 2 / L'esprit de la lettre, Études réunies et présentées par Nadine Ly, Maison des Pays Ibériques (GRIAL), Presses Universitaires de Bordeaux, 1992, p. 151-180. L'expression citée se trouve à la page 161.

2. Ibid., p. 158.

3. L'édition critique consultée est : Lope de Vega, Rimas 1/ [Doscientos sonetos], Felipe B. Pedraza Jiménez éd., Université de Castilla-La Mancha, Servicio de Publicaciones, 1993. Le lecteur peut également se référer à l'article de G. I. Brown, "The peregrino amoroso: Four moments of poetic configuration in the spanish love sonnet ", Neophilologus, Groningen, 1976, vol. 60, n 3, p. 376-388.

4. F. P. Jiménez, dans son édition critique, est sensible au sonnet 19, « uno de los poemas más 
Avant d'aborder ce premier texte, il reste à essayer de justifier l'adjectif polémique de "gothique " dont j'ai osé qualifier l'inspiration poétique de Lope. Il ne s'est pas seulement agi pour moi de m'adonner aux délices interdites de l'anachronisme. L'application de ce terme d'architecture à la littérature a été parfaitement datée par Maurice Lévy dans son ouvrage de référence ${ }^{5}$, le genre naissant en 1764 avec le roman anglais Le Château d'Otrante. Mais le spécialiste lui-même admet le rôle éminent joué par Dante, Shakespeare ou le roman de chevalerie dans le façonnement d'une poétique qui englobe, outre les châteaux et les couvents du " genre noir ", les forêts impénétrables, les mausolées et les cimetières, les tempêtes en mer, l'apparition des spectres.

La présence d'un imaginaire « médiéval » aux côtés de sources gréco-latines et humanistes dans le substrat de la littérature espagnole auriséculaire est trop connue pour que l'on y revienne. Peut-être n'est-on pas allé assez loin dans l'identification de certaines œuvres "pré-gothiques », même si Fernando Rodríguez de la Flor ${ }^{6}$ ou les spécialistes des nouvelles « ténébristes » de María de Zayas ont déjà exploré le " cerveau noir ${ }^{7}$ " de certains textes. M. Lévy écrit, du reste : "Comme le baroque incite à la fantaisie et aux jeux de surface, le "gothique" élève - ou entraîne vers d'abyssales profondeurs ${ }^{8}$ ". Je vais m'efforcer de prouver que le poème n'est pas qu'une variation de plus à partir du motif ressassé du pèlerin amoureux, qu'il est parcouru d'une terreur authentique, et qu'il cerne, comme le fera la littérature gothique, cette "manière [...] de dire l'Autre Chose ? La précarité, l'abîme et le manque ${ }^{9} "$.

Passando un valle escuro al fin del día,
tal que jamás, para su pie dorado,
el sol hizo tapete de su prado,
llantos crecieron la tristeza mía.

Entrando, en fin, por una selva fría, vi un túmulo de adelfas coronado,

inquietantes de las Rimas, que la critica no ha sabido valorar adecuadamente ", op. cit., p. 226. 5. Maurice Lévy, Le Roman " gothique » anglais (1764-1824), Bibliothèque de « L'Évolution de l'Humanité ", Albin Michel, 1995.

6. Fernando Rodríguez de la Flor, «La Peninsula metafisica : arte, literatura y pensamiento en la España de la Contrarreforma ", Era melancólica : Figuras del Imaginario Barroco, Madrid, Biblioteca nueva, 1999.

7. J'emprunte cette expression à Maria Zerari.

8. M. Lévy, op. cit., p. 8.

9. Ibid., p. XXV. 
y un cuerpo en él, vestido aunque mojado, con una tabla en que del mar salía.

Dixome un viejo de dolor cubierto:
"Este es un muerto vivo (jestraño caso!),
anda en el mar, y nunca toma puerto".

Como vi que era yo, detuve el passo : que aun no me quise ver después de muerto, por no acordarme del dolor que passo.

Mon examen du texte s'appuiera sur la méthode de l'analyse littérale dont Nadine Ly a énoncé de manière définitive les enjeux théoriques. À l'appui de cette démarche, je ferai également référence à un article célèbre ${ }^{10}$ où Gérard Genette propose de comprendre la figure rhétorique comme une fiction en germe ou en miniature :

Une figure est (déjà) une petite fiction, en ce double sens qu'elle tient généralement en peu de mots, voire en un seul, et que son caractère fictionnel est en quelque sorte atténué par l'exiguïté de son véhicule et, souvent, par la fréquence de son emploi, qui empêchent de percevoir la hardiesse de son motif sémantique : seuls l'usage et la convention nous font accepter comme banale une métaphore comme " déclarer sa flamme ", une métonymie comme "boire un verre », ou une hyperbole comme " mort de rire ". [...] une fiction n'est en somme qu'une figure prise à la lettre et traitée comme un événement effectif ${ }^{11}$. (Nous soulignons).

Pour ma part, je vais considérer l'oxymore majeur du sonnet ( $u n$ muerto vivo", vers 10) non pas uniquement comme une formule stylistique héritée $\mathrm{du}$ cancionero pour évoquer de manière hyperbolique la souffrance amoureuse du poète, mais comme le point d'ancrage, pris par moi " au sérieux ${ }^{12}$ ", d'une fiction - le poème. Ce franchissement de la frontière habituellement tracée entre poésie lyrique et récit m’entraînera, dans la seconde partie de mon exposé, du côté du théâtre, un " milieu de fiction " amplifié où la figure du muerto vivo (un "énoncé physiquement impossible ${ }^{13} »$ ), exploite son potentiel de personnage dramatique. Je me dispose par conséquent à observer

10. Gérard Genette, Métalepse / De la figure à la fiction, Collection " Poétique ", Éditions du Seuil, 2004.

11. Ibid., p. 17.

12. Ibid., p. 20.

13. Ibid., p. 17. 
la métamorphose d'une figure de l'esprit en matière "romanesque " - et même, pour citer toujours G. Genette, en une " fiction de type fantastique, ou merveilleux ${ }^{14}$ ". Ce " régime fantastique ${ }^{15}$ " justifierait les pouvoirs de suggestion du poème, au-delà des jeux conceptistes ou des réécritures pétrarquisantes.

Le sonnet se présente sous la forme d'un récit énigmatique, le poète faisant, au milieu d'une forêt, la découverte d'un tumulus montrant un cadavre dont l'apparence est celle d'un naufragé encore trempé et amarré à une planche qui l'a empêché de sombrer. La résolution (si résolution il y a) de l'énigme s'effectue en deux temps :

- dans le premier tercet, un vieillard, témoin de la scène, qualifie la créature de "mort-vivant", perdu en mer, errant sans fin ;

- dans le second tercet, le poète dévoile l'identité du mort-vivant ; il s'agit de lui-même, une vision qu'il fuit pour ne pas aggraver sa douleur.

Le vers 10 pourrait bien être significatif du passage de la figure à la fiction, puisque le mort-vivant y trouve son vrai statut de personnage, inscrit dans un contexte spatio-temporel étrange, certes, mais effectif : estraño caso. Caso rend, me semble-t-il, insuffisantes, même si elles sont, évidemment, en grande affinité avec le sonnet, les interprétations uniquement symboliques et allégoriques : chemin de la vie ; vieillard du Desengaño ; souffrances infinies de l'errement amoureux ; océan de dangers - le pèlerin d'amour prenant les contours d'un Sysiphe. Or, l'amour, par exemple, est littéralement absent du sonnet, même si les commentateurs le considèrent forcément implicite.

Le caso dont le vieil homme est l'informateur déborde par ailleurs largement des limites du tercet, si l'on s'intéresse d'emblée au tumulus forestier du second quatrain, et à l'incompréhensible présence, en un lieu fixe et immuable, dressé pour l'éternité, d'un corps dont il est précisé qu'il ne saurait être qu'en mouvement. L'impossible jonction entre le monticule de terre ou de pierres et la mer qui sert de cadre aux dérives de l'éternel naufragé se produit pourtant. Un contenant inapproprié - car sec, vertical et solide, accueille un contenu humain inconcevable en dehors d'un milieu aqueux, horizontal, illimité - la mer, deux fois nommée. Le poème touche à l'irreprésentable tout en s'appliquant avec précision à décrire ce théâtre funèbre.

14. Ibid., p. 25.

15. "Le scandale, de toute évidence, tient précisément à cette littéralisation de ce qui n'était, pour les esprits sages, qu'une plaisante façon de parler, et qui devient une manière d'être, d'occuper l'espace et de passer le temps. » Ibid., p. 31-32. 
Tel une impossible didascalie, le quatrain détaille le corps vêtu quoique trempé d'eau salée, en contact étroit avec une planche directement issue de la mer. Le sonnet assume tranquillement, presque familièrement, son incongruité. L'immersion dans l'eau devrait renvoyer à la nudité corporelle - l'idée des vêtements abîmés apparaissant comme particulièrement redoutable. Je rappelle, par exemple, ce qu'écrit le dramaturge dans la dédicace à La Viuda valenciana : la gala de nadar es saber guardar la ropa. Cet homme vestido aunque mojado est donc bien une vision d'horreur, malgré le vers 8 (con una tabla en que del mar salía), apparemment consacré à un naufragé sauvé des eaux par une planche. Cette image fort proche de celle de la naissance (salir del mar) a été préalablement "plombée " (si l'on ose dire) par celle du promontoire macabre faisant office d'île-tombeau. L'Ulysse lopesque n'est pas plus sauf sur terre que sur mer, quand il est ballotté par les flots ou échoué sur la rive.

Construit sur le modèle du " mort-vivant ", le personnage " habillémouillé " est celui qui n'a pas su garder intacte son enveloppe textile; il l'a gâtée, comme sera gâté - liquéfié à son tour - le corps mortel qu'elle revêt. Couvert de deux matières (l'eau salée; le tissu) qui s'excluent l'une l'autre, le noyé a perdu sa barrière protectrice : la limite entre les règnes, entre les éléments, les états physiques et mentaux, la mort et la vie s'est dissoute. Le paradoxe du naufragé amphibie met l'accent sur un phénomène de redondance et de superposition permettant d'appréhender la structure fictionnelle du poème.

En effet, la ligne du récit respecte l'ordre du voyage, du déplacement d'un espace à un autre, en l'occurrence d'une obscure vallée jusqu'à la forêt froide où se dresse le tertre. Le défilé des paysages préserve la successivité des séquences, celle des lieux et celle du temps, qui s'écoule également jusqu’à ce fin del día marquant bien le passage d'un moment à un autre. Il n'est donc pas fortuit que les premiers termes des quatrains soient passando et entrando...por. L'impulsion qu'ils donnent au sonnet installe une fluidité narrative évocatrice d'on ne sait quelle avancée ou aventure à venir. Or le mouvement du sujet et du texte est concurrencé, contredit, par tout un lexique de l'épaisseur et de la profondeur, renvoyant à des objets stratifiés ou à des substances superposées :

- vers 3 : el sol hizo tapete de su prado, pour le tapis de fleurs qui recouvre la vallée ;

- vers 4 : llantos crecieron la tristeza mía : après tapar, crecer; les sanglots s'ajoutent à la tristesse, comme sous l'effet d'une crue des eaux ; 
- vers 6 : un túmulo de adelfas coronado : après tapar et crecer, coronar; la couronne de lauriers-roses vénéneux est posée au sommet du tombeau.

Les trois termes en $t$ (tapete, túmulo, puis tabla) se renforcent l'un l'autre, la tabla ayant pour fonction d'offrir au corps du naufragé une couche isomorphe.

Dès lors, la dynamique horizontale parât «naufrager » à son tour : salir del mar (vers 8), nunca tomar puerto (vers 11), detener el passo (vers 12) - les trois ensembles dissipent l'illusion du trajet d'un point à un autre ou d'un terme fixe à l'issue d'un processus mesurable. Linvasion d'une écriture de la surimpression - êtres et éléments collés entre eux - renverse l'ordre de la Nature et de la Vie. Des paysages qui ne sauraient se côtoyer fusionnent. Selva et mar sont confondus dans le second quatrain, leur mutuelle froideur ayant absous toute limite. Quant à ce crépuscule auquel l'on avait cru au vers 1 , il est contesté par l'hyperbole des vers 2 et 3, la vallée étant obscure non pas parce que le soleil se couche, mais parce que les parois qui enclosent la plaine empêchent toute incursion de la lumière. L'ensemble escuro al fin del día constitue un exemple d'agglutination spatio-temporelle. Les plans glissent les uns sous les autres, creusant une perspective verticale, celle de l'enlisement, de l'ensevelissement.

Le tumulus lui-même, sorte de version "gothique " de la pyramide égyptienne, s'élève au second quatrain comme s'il emplissait le vide abrupt, triangulaire, laissé dans le premier quatrain par les contours de la vallée obscure. Cette esthétique des deux moitiés - le tumulus comme $V$ renversé - $s^{\prime}$ inscrit parfaitement dans la fiction macabre du poème. Le sonnet décrit l'Autre Monde, l'autre Moitié du Monde, celle où les fleurs ne composent pas des tapis parfumés mais des couronnes funéraires.

Mais la création de cet univers parallèle ne s'en tient pas qu'à des effets de symétrie de signe contraire. Dans cet autre monde qu'est le sonnet, la viscosité des images l'emporte sur la netteté des contrastes. Le vieillard du premier tercet est, à la lettre, recouvert de douleur - couvert d'une couche d'humidité poisseuse (larmes ou eau de mer) qui fait de lui l'un des trois visages du mort-vivant déplié/décliné en trois épaisseurs d'expérience. Par sa vieillesse, le personnage est à cheval entre la vie et la mort, comme le naufragé ballotté par les eaux, comme le visiteur du Royaume des Ombres. Si le poète reconnaît son corps exposé - un cuerpo en él vestido aunque mojado - c'est qu'il est peut-être l'âme errante et en peine de ce cadavre sans repos. D'où le verbe andar du vers 11 - anagramme de nadar - mais surtout le verbe 
réservé aux spectres et aux esprits, si l'on en croit ceux qu'invoquent, par exemple, les personnages de la comedia Los Muertos vivos ${ }^{16}$ :

\author{
que si es alma, no por buena \\ anda en pena por ahi $[. .$. \\ Quedo, que si es cosa mala, \\ en asiéndola, resbala.
}

Un tel imaginaire de la matière soumet l'écriture à un traitement radical : plutôt que de construire un espace de transfiguration, le poème montre des métamorphoses arrêtées en leur milieu - ce lieu ou ce moment inimaginables où les choses tiennent encore de leur double nature - : un mort vivant, la mer allée avec la forêt, une planche à mi-chemin entre le radeau et la bière. Cette modalité de jonction contre-nature, de superposition ou d'adhérence, donne un aperçu de ce qu'implique la mort, envisagée comme un impossible passage. La démultiplication du sujet en au moins trois instances simultanées résulte de l'imbrication des niveaux : plusieurs états de douleur, plusieurs époques et plusieurs cadres s'incorporent dans une fiction dont le récit est endigué, s'étale et s'amoncelle indéfiniment sur lui-même.

Il reste à observer ce qu'il advient dans une pièce de théâtre fameuse, qui pourrait bien être l'amplification de cet embryon de fiction que constitue tout particulièrement le dernier tercet du sonnet, "piégé " par la viscosité du temps et de l'identité. Si l'on veut bien les lire comme un estraño caso, ces trois vers résument l'essentiel du dénouement de El Caballero de Olmedo ${ }^{17}$.

Il est, de mon point de vue, possible de mettre en relation étroite le sonnet 19 et l'histoire tragique du Chevalier. La pièce instaure en effet une poétique de la fusion des plans ${ }^{18}$, comme dans le célèbre récit du rêve aux oiseaux à l'Acte 2, où don Alonso narre à son valet Tello unos sueños que he tenido ${ }^{19}$. Or, le Chevalier détaille la vision qu'il a eue en sortant de son lit au petit jour pour regarder son jardin par la fenêtre. Il raconte ce qu'il a vu comme

16. Lope de Vega, Comedias, VII, Biblioteca Castro, Turner, Madrid, 1994, p. 697.

17. Date de composition : autour de 1620. L'édition consultée est celle de Federico Carlos Sáinz de Robles, Lope Félix de Vega Carpio, Obras Escogidas, Tomo 1, Teatro, 1, Madrid, Aguilar, $5^{a}$ edición, 1990.

18. Dans le tercet annonciateur de la tragi-comédie, la viscosité du temps était déjà manifeste, le "présent " du récit coupé net (detuve el paso) s'agglutinant à la perspective future (después de muerto) et au passé (no acordarme). L'audace du poète est grande, lorsqu'il relate qu'il se découvre après sa mort et se souvient de son présent. Rien d'étonnant à cela, il est vrai, s'il existe un état intermédiaire qui tient de l'avant et de l'après.

19. Op. cit., p. 813. 
s'il l'avait rêvé, ou ce qu'il a rêvé comme s'il l'avait vu. Don Alonso est-il, en profondeur, un dormeur somnambule, déambulant tout au long de la pièce dans un état autre que celui de veille - un état que l'on va s'employer à mieux définir?

Aussi bien le poème que la tragi-comédie du Chevalier d'Olmedo s'appuient sur le motif légendaire bien connu de la rencontre avec le double, comme signe avant-coureur de la mort. Ce substrat inclut, par ailleurs, l'anecdote du jeune homme qui voit passer son propre enterrement - une anecdote terrifiante dont on perçoit certains échos dans une autre ouvre " gothicisante " ô combien étudiée : El Burlador de Sevilla y Convidado de piedra. La confrontation effrayante du sonnet 19 se produit en deux temps : une perception auditive d'abord (llantos crecieron la tristeza mía) précédant la découverte visuelle (vi un túmulo [...] y un cuerpo en él; vi que era yo).

Dans El Caballero de Olmedo, don Alonso évolue dans un paysage très proche de celui du poème ; la présence des arbres, de l'obscurité et du froid, enveloppe le voyageur qui insiste, comme dans le sonnet, sur l'absence angoissante du soleil :

$$
\begin{aligned}
& \text { ¿Qué escuridad! Todo es } \\
& \text { horror, hasta que el Aurora } \\
& \text { en las alfombras de Flora } \\
& \text { ponga los dorados pies }{ }^{20} \text {. }
\end{aligned}
$$

Don Alonso, cependant, a déjà été sollicité en quittant Medina par l'apparition d'une Ombre menaçante :

(Al entrar, una sombra con una máscara negra y sombrero, y puesta la mano en el puño de la espada, se le ponga delante.) [...]

$\begin{array}{ll}\text { Alonso : } & \text { ¿No dice } \\ & \text { quién es? } \\ \text { Sombra : } & \text { Don Alonso. } \\ \text { Alonso : } & \text { ¿Cómo? } \\ \text { Sombra : } & \text { Don Alonso. } \\ \text { Alonso : } & \text { No es posible. } \\ & \text { Mas otro será, que yo } \\ & \text { soy don Alonso Manrique }{ }^{21} .\end{array}$

Comme au vers $13 \mathrm{du}$ sonnet (aun no me quise ver), le personnage refuse de se reconnaître. Mais une présence saisissante, auditive cette fois, devient 
perceptible au milieu du chemin qui sépare Medina d'Olmedo, il s'agit de celle du célèbre " couplet » :

\author{
Que de noche le mataron \\ al Caballero, \\ la gala de Medina, \\ la flor de Olmedo 22 .
}

La chanson a été précédée, il faut le noter, par les murmures inquiétants que la nature exhale quand elle est plongée dans la nuit. Comme dans le poème, ces sons plaintifs accroissent la peine du sujet :
Del agua el manso rüido
$y$ el ligero movimiento
destas ramas con el viento
mi tristeza aumentan más ${ }^{23}$.

Le même effet d'épaississement de la tristesse s'exprime lorsque don Alonso entend le chant du laboureur :

\title{
¡Qué mal la música sabe si está triste el pensamiento! ${ }^{24}$
}

Cette superposition de deux complaintes, celle du couplet et celle du Chevalier accablé de solitude, rappelle le phénomène du sonnet. Dans la séguedille, don Alonso n'est plus chevalier d'Olmedo, mais ornement de Medina, fleur d'Olmedo : les deux espaces se sont collés l'un à l'autre, le personnage, comme un liquide, ayant débordé, s'étant répandu sur les deux rives de l'arroyo médian. La rencontre du chevalier errant avec les échos de sa propre mort produit non pas une unification du sujet mais un trop plein, une nouvelle redondance :

$\begin{array}{ll}\text { Alonso : } & \text { A mi suelen llamar } \\ & \text { el Caballero de Olmedo } \\ & \text { y yo estoy vivo. }[. . .]\end{array}$

22. Ibid., p. 820.

23. Ibid., p. 820.

24. Ibid., p. 820. 


$\begin{array}{ll}\text { Labrador : } & \text { No puedo } \\ & \text { deciros deste cantar } \\ & \text { más historia ni ocasión } \\ & \text { de que a una Fabia la oi }{ }^{25} .\end{array}$

Nommément comparée à Circé au troisième acte ${ }^{26}$, l'entremetteuse magicienne est à associer à la fatalité de la noyade en mer rappelée par le Chevalier à l'Acte 2 quand il se compare à Léandre traversant l'Hellespont à la nage ${ }^{27}$. Les allusions du texte dramatique au Royaume des Morts ${ }^{28}$ assimilent le «naufrage » terrestre du Chevalier à la descente aux Enfers de héros tels qu'Énée ou Ulysse. Lope de Vega agglutine les deux trajectoires, l'horizontale maritime et la verticale souterraine dans le sens d'un effacement des lignes de démarcation.

La fiction surnaturelle que risque le dramaturge comme l'avait osée le poète dans le sonnet 19 , c'est celle d'un personnage mort, qui ignore qu'il est mort, et qui ne comprend pas pourquoi il n'est pas à sa place parmi les vivants. Les preuves sont pourtant là. Le texte signifie maintes fois au héros qu'il n'est plus de ce monde : l'apparition masquée, lui tend, par exemple, un miroir, lui permettant de se voir tel qu'il est, un fantôme.

Ainsi, je suggère qu'une autre interprétation s'ajoute à celles, plus vraisemblables, mais moins logiques, qui existent déjà : une interprétation "gothique ". La pièce conte les aventures d'un habitant de l'au-delà, lequel, frappé d'amnésie (por no acordarse del dolor que passa), se croit en vie, malgré les indices dont il dispose pour se savoir défunt ${ }^{29}$. Le passage, à mon avis, le moins souvent commenté est celui extrait du célèbre romancillo du deuxième acte, où don Alonso se déclare incapable de posséder physiquement doña Inés, pourtant consentante:

25. Ibid., p. 821.

26. "No supo Circe, Medeal ni Hécate lo que ella sabe» (Ibid., p. 815).

27. «Leandro pasaba un marl todas las noches, por ver/ si le podía beber/ para poderse templar; I pues si entre Olmedo y Medinal no hay, Tello, un mar ¿qué me debel Inés?» (Ibid., p. 803).

28. "Fabia, que puede detener un riol y en los negros ministros de Aquerontel tiene, como en vasallos, señorio ;/ Fabia, que deste mar, deste horizonte, / al abrasado clima, al norte friol puede llevar un hombre por el aire [...]» (Ibid., p. 820). Au pèlerinage amoureux se mêle, dans la tragi-comédie, la volonté obsessionnelle de don Alonso de rejoindre ses parents à Olmedo en pleine nuit, malgré le danger imminent ; cette hantise rapproche le personnage lopesque d'Énée, retrouvant son père Anchise dans l'Autre Monde.

29. Certes, les poèmes qu'il compose pour dońa Inés le présentent mourant d'amour dans la tradition du cancionero, mais certains vers peuvent être lus "sérieusement ", lorsqu'ils décrivent le statut spectral du héros: Parto a morir, y te escribol mi muerte, si ausente vivo ; porque tengo, Inés, por ciertol que si vuelvo será muertol pues partir no puedo vivo (Ibid., p. 818). 


\author{
Llorando por mi ausencia \\ Inés quedó aquel día, \\ que sus lágrimas fueron \\ de sus palabras firma. \\ Bien sabe aquella noche \\ que pudiera ser mía. \\ Cobarde amor ¿qué aguardas, \\ cuando respetos miras ${ }^{30}$ ?
}

Décomposée en plusieurs états d'elle-même qui s'agglutinent dans le texte, l'ombre vaine ne peut s'accoupler à une femme vivante.

L'on sait que Lope de Vega ignorait tout du fait divers tragique survenu sous le règne de Charles-Quint à un certain don Juan de Vivero ${ }^{31}$ entre Olmedo et Medina. Le poète n'aurait eu connaissance que de la copla funèbre et sans doute d'un ballet, fort éloigné des sources historiques, contant la mort du Chevalier. C'est bien d'un héros mort, célèbre seulement pour sa mort, que le dramaturge a hérité. Pour en faire un galant de théâtre, il s'est ingénié à lui donner une existence scénique, mais l'écriture de la pièce porte les traces d'une métamorphose comme enlisée ou suspendue en son milieu. La transformation du chevalier mort en galant bien vivant ne s'est pas opérée complètement. Le personnage reste lesté de toute la morbidité de son passé poétique de cadavre, et don Alonso n'est devenu, dans le processus de création, qu'une sorte de larva enveloppée dans les oripeaux d'un vivant.

Le génie de Lope consiste à ne pas avoir allégé son personnage de sa propre mort déjà survenue - de l'avoir intégrée pour faire de son héros un revenant. D’où les vers de Tello à doña Inés :

\author{
Oye una glosa a un estribo \\ que compuso don Alonso \\ a manera de responso \\ si los hay en muerto vivo ${ }^{32}$.
}

Certes, Lope de Vega met en scène au troisième acte, quasiment en direct, la mort du Chevalier assassiné à l'arme à feu par don Rodrigo et ses

30. Ibid., p. 812.

31. Les informations concernant le "fait divers " tragique sont consignées dans l'introduction que Francisco Rico a consacrée à son édition de la célèbre pièce, Cátedra, Letras Hispánicas, Madrid, 1992, p. 36.

32. Op. cit., p. 805. La glose rend bien l'idée d'une matière visqueuse qui déborde de la strophe initiale, et qui exprime rigoureusement le labeur dramatique - une amplification qui confond ses contours avec ceux du couplet d'origine, en l'enrobant de matière textuelle. 
complices. Mais je crois possible d'avancer que cette exécution n'est qu'une mort de plus - et non la mort de don Alonso - non pas un matar mais un rematar, l'entreprise poétique du dramaturge consistant, strictement, à tuer un mort.

Il m'est apparu suggestif, pour conclure, de souligner que cette appréhension des états intermédiaires, indécidables ou visqueux - déjà fortement présente dans la poésie lyrique des années 1600 - témoigne d'une réécriture incessante des modèles mythiques : la création équivaut, de ce fait, à une adjonction de substance textuelle. Ce sens fécond des sédimentations n'engendre pas que des chimères ou des monstres précurseurs du fantastique - comme ce " mort-vivant " auquel l'on a tenté de rendre sa consistance dramatique. Pour emprunter à Freud l'expression qu'il emploie dans L'inquiétante étrangeté 33 , le dramaturge espagnol, conscient de produire des œuvres hantées, a su identifier mais également traduire en termes de fiction, le « caractère démonique » du labeur littéraire. 